\title{
Effectiveness of Cervical Stabilisation Training and Correction of Muscle Imbalance, following reduction of Atlanto-Axial Rotatory Subluxation: A single case study
}

\begin{abstract}
Atlanto-axial rotatory subluxation is a rare, controversial and frequently misdiagnosed condition occurring primarily in children. A single case study design was DEBBIE DAVIDSON used to evaluate the effectiveness of cervical stabilisation training and correction of muscle imbalance, following reduction of this condition, in a nine year old boy. The study was conducted over a six week period during which the subject maintained a daily diary to record his symptoms. The programme consisted of various muscle relaxation and lengthening techniques, endurance training of the deep cervical flexors and lower scapular stabilisers, as well as postural re-education. No treatment was directed at the articular component. The patients signs and symptoms were greatly reduced during the study period. This study supports the importance of correcting the muscle dysfunction component in cervicogenic pain sufferers.
\end{abstract}

\section{INTRODUCTION}

Atlanto-axial rotatory subluxation is a rare, poorly understood condition that occurs primarily in children (Fielding \& Hawkins, 1977; Phillips et al, 1989; Robert \& Hensinger, 1993). The subluxation occurs when the lateral mass of the atlas rotates forward on the facet of the axis when turning the head and remains fixed in this position on retuming the head to neutral (Henrys et al, 1977). Fielding \& Hawkins (1977) classified rotatory subluxation into four types according to the degree of anterior instability. An illustrated case in each classification is demonstrated below (fig 1).

Although the rotatory subluxation is usually short-lived and easily correctable (Phillips et al, 1989; Robert \& Hensinger, 1993), Fielding \& Hawkins (1977) described a series of patients in whom the diagnosis was made late and the subluxation was irreducible. They termed this outcome rotatory fixation and recommended atlanto-axial arthrodesis for relief of symptoms.

The onset is usually associated with an upper respiratory tract infection (URTI) or minor trauma (Fielding \& Hawkins, 1977; Phillips et al, 1989; Henrys et al, 1978). The exact aetiology of the condition is unknown, but is thought to be due to increased laxity or deficiency of the alar and transverse ligaments and of the capsular structures secondary to inflammation, infection or trauma (Phillips et al,

CORRESPONDENCE:

Debbie Davidson (Shuter)

BSc (Physiotherapy) Witwatersrand

Suite 168, Postnet XII, Craighall 2024

(H) 011-880-6979

(W) $011-442-8233$

(C) 083-457-7558
1989; Dvorak \& Panjabi, 1987; Roach et al, 1984; Aspinall, 1990).

The patient presents with a torticollis deformity which is typically likened to a robin listening for a worm, the so-called "cocked robin" position (Fielding \& Hawkins, 1977; Robert \& Hensinger, 1993; Phillips et al, 1989). Unlike patients who have muscular torticollis, the patient presents with spasm in the stemocleidomastoid muscle on the side to which the chin is rotated, suggesting an attempt to reduce the deformity (Phillips et al, 1989). Diagnosis is primarily dependant on history, clinical findings and dynamic computed tomography (Fielding et al, 1978).

Whilst the condition exists, atlantoaxial stability may be compromised and even minor injury to the neck may produce catastrophic results including spinal cord compression and brainstem ischaemia (Phillips et al, 1989; Robert \&
Hensinger, 1993; Henrys et al, 1977).

It is well documented that trauma to the cervical spine is one of the most commonly cited provocative causes of headache (Beeton \& Jull, 1994; Bogduk, 1986; Edeling, 1982; Jull 1988). This is supported by the frequent accounts of frontal and occipital headaches following reduction of the subluxation, that were found hidden within the case reports of the literature. It is postulated that these headaches arise from the ligaments and the atlanto-axial joints that are exposed to excessive mechanical stress as a result of the subluxation (Fielding \& Hawkins, 1977; Phillips et al, 1989; Robert \& Hensinger, 1993). No literature regarding physiotherapy management following reduction could be found.

Studies by Watson (1990) have shown that cervicogenic headache sufferers demonstrate weakness and loss of endurance of the deep cervical flexors

\section{Figure 1.}

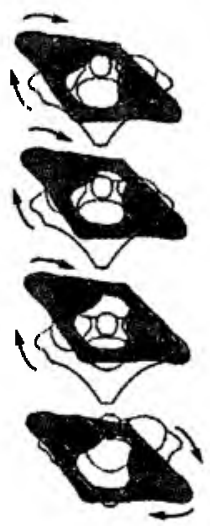

Type I - rotatory subluxation with no anterior displacement.

Type II - rotatory subluxation with anterior displacement of three to five millimetres.

Type III - rotatory subluxation with anterior displacement of more than five millimetres.

Type IV - rotatory subluxation with posterior displacement. 
and often exhibit a forward head posture as well as striking muscle imbalance of the 'proximal crossed syndrome' type described by Janda (1988). Clinical treatment of muscle imbalance using cervical stabilisation training is giving very encouraging long term results in patients with chronic cervical pain syndromes (Beeton \& Jull, 1994; Watson, 1990; Jull et al, 1995).

On the basis of the above information, this study was designed to:

a) Describe this rare condition to other physiotherapists and highlight the importance of prompt recognition and referral. b) To determine if cervical stabilisation training and correction of muscle imbalance would alter the patient's signs and symptoms following reduction of atlantoaxial rotatory subluxation.

\section{CASE REPORT}

\section{INITIAL EXAMINATION}

\section{Subjective Assessment}

A nine year old boy, actively involved in sports, presented with severe left sided neck pain and continuous headache associated with dizzy spells. He described his pain as "vicious" in the nape of his neck, extending as a "dull, dizzy" pain over the top of his head. He had fallen whilst waterskiing two days previously and noticed slight pain on the left side of his neck. He awoke the following morning with a typical torticollis.

Four years previously he fell from a 2 metre high wall onto his head, landing on a concrete surface. He sustained a left frontal skull fracture and a chronic subdural haemorrhage. According to the patient's mother he had since complained of no related symptoms.

No medical practitioner had been seen for this condition and no $\mathrm{X}$-rays had been taken.

\section{Objective Assessment}

The fact that there were no X-rays, no doctor's referral and a history of severe previous trauma indicated the need for extreme caution to be taken in examination.

On observation, the patient had a severe torticollis with lateral flexion to the left, rotation to the right and slight flexion (Figure 2). The right sternocleidomastoid muscle appeared to be in severe spasm.

Due to the fact that the patient was in acute pain and unwilling to move his neck, the objective assessment was limited to a neurological examination, that essentially revealed no abnormalities. He was referred urgently to an orthopaedic surgeon.

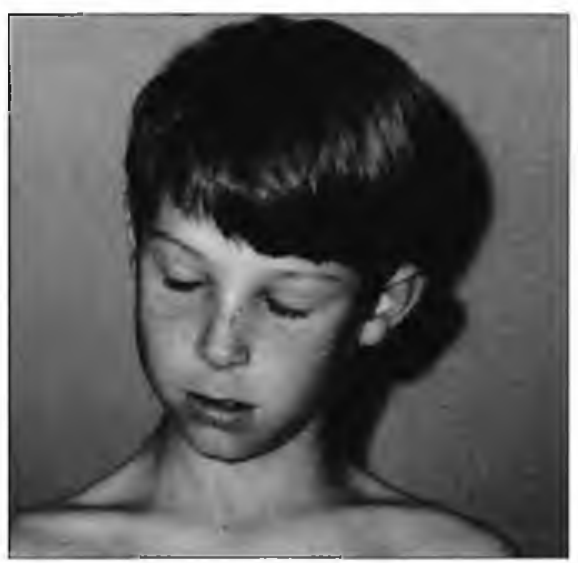

Figure 2. Typical "cocked robin" position of rotatory subluxation

The patient was diagnosed as having a type II atlanto-axial rotatory subluxation which was confirmed with dynamic computed tomography. Reduction of the subluxation was achieved with bedrest, antiinflammatories and strong muscle relaxant medication, followed by immobilisation in a rigid cervical brace for ten weeks to allow for ligamentous healing.

\section{EXAMINATION FOLLOWING REDUCTION AND IMMOBILISATION}

\section{Subjective assessment}

The patient now described three distinct intermittent symptoms which started following removal of the cervical brace three weeks previously (Figure 3).

Symptom 1 - was aggravated by sustained postures and occurred mainly at school, playing on the computer or whilst watching TV, lasting anything from two hours to the whole day.

Symptom 2 - occurred with awkward quick movements involving extreme flexion, for example, removing a T-shirt over his head. The pain only lasted for a "split" second.

Symptom 3 - was aggravated by anything that stretched the left side of his neck, for example turning to the right, serving at tennis and the end of his golf swing. The pain only lasted a few min- utes.

He also commented that his head felt "too heavy for his neck" and his mother added that he frequently supported his head with his left hand, especially when watching TV or doing homework. A soft collar was to be worn at specified risky times during the day and participation in contact sports was not allowed.

\section{Objective assessment}

On observation the patient showed postural deformities of increased thoracic kyphosis, rounded shoulders and a severe "poking chin". There was noticeable scapular winging bilaterally. The neck appeared to be held in slight lateral flexion to the left, and shortening and spasm was noted in the left scalenes and upper trapezius area.

Both rotation and lateral flexion to the right were limited to half range reproducing symptom three. Cervical flexion was limited to five centimetres from chin to sternum also reproducing symptom three. Cervical extension and left rotation and lateral flexion were full range and pain free with gentle overpressure.

Symptom 1 was not reproduced as it occurred following greater than half-anhour of a sustained posture. It was also deemed unnecessary to reproduce symptom 2 due to its severity, and the nature of the condition.

Neural tension tests and the neurological examination were unremarkable. The vertebral artery tests were not completed due to limitation of the patient's pain free range of motion.

On palpation active trigger points, protective spasm and shortening were found in the left scalenes, levator scapulae, upper fibres of trapezius (UFT) and the suboccipital muscles. Gentle pressure applied to left first rib posteriorly reproduced symptom 3. Central, left and right unilateral postero-anterior movements of the second cervical vertebra produced sharp local increases in pain.

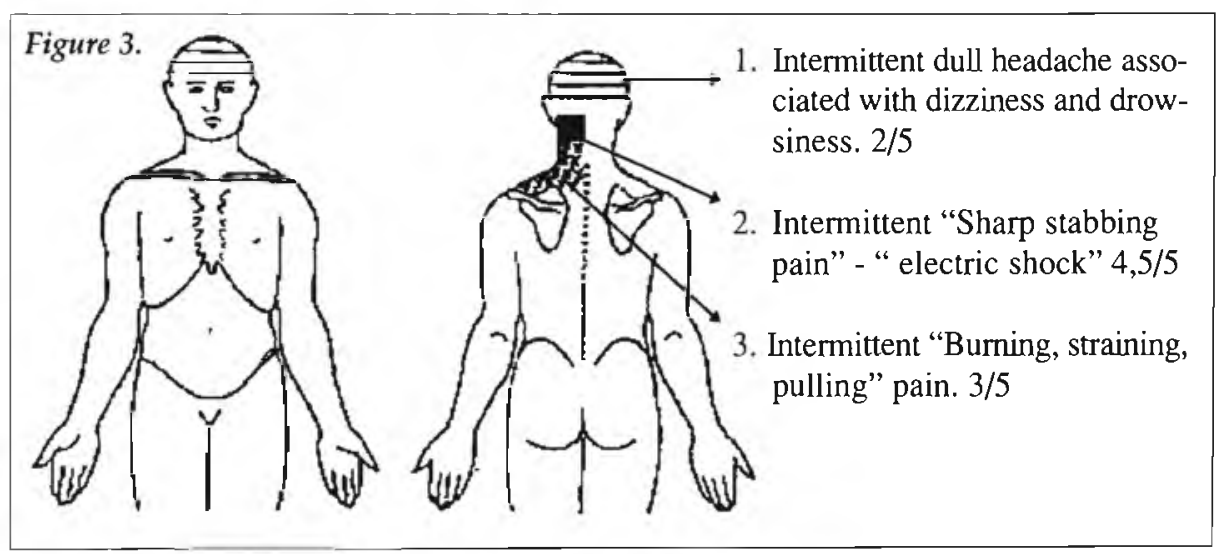




\section{TREATMENT}

The study was carried out for six weeks, during which the subject, with the help of his mother, maintained a daily diary to record his symptoms. Information documented included the frequency, duration, intensity and any known causative factor for the symptoms.

The endurance capacity of the deep cervical flexors (DCF) was measured as the number of times the patient could hold a chin tuck position for ten seconds in the supine position using a biofeedback pressure sensor (Figure 4). Similarly the lower scapular stabilisers (LSS) were assessed by the number of times the patient could hold a static inner range contraction for ten seconds in the standard muscle test position. These are the standard clinical tests described by Beeton \& Jull (1994) who regarded an endurance limit of a minimum of ten 10 second holds as a normal endurance level. The tests were terminated when the subject was either unable to maintain the contraction or substitution strategies by other muscles were observed.

Treatment was aimed at restoring muscle balance.

\section{Weeks I and 2}

The patient's endurance capacity measurements of the:

a) DCF was four 10-second holds increasing the pressure in the cuff from 20 to $28 \mathrm{~mm} \mathrm{Hg}$ before substitution strategies by the sternocleidomastoid muscles was observed.

b) LSS was five 10-second holds before loss of control of the scapular position was observed.

The patient attended physiotherapy sessions twice weekly. A moist hot pack was applied for fifteen minutes. Following this, gentle muscle lengthening techniques including stretching, trigger point therapy and myofascial release were applied to the left LFT, scalenes, levator scapulae and the suboccipital extensors.

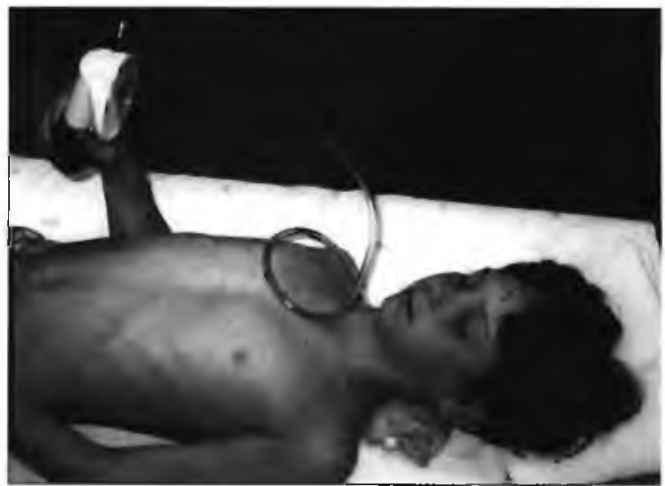

Figure 4. Assessing endurance of deep cervical flexors using pressure sensor.
He was to perform a home programme twice daily which included:

a) four gentle muscle stretching exercises for the left UFT, scalenes, levator scapulae and the suboccipital extensors. Each stretch was to be done three times and held for thirty seconds.

b) five 10 -second holds using the chin tuck action, with the assistance of feedback from the stabiliser.

c) five 10 -second static contractions of the LSS.

\section{Weeks 3 and 4}

Cervical rotation and lateral flexion to the right were greatly improved. They were full range reproducing symptom 3 only at the end of range. Cervical flexion was full range and pain free with gentle overpressure. The subject's endurance capacity of the:

a) DCF improved to seven 10-second holds.

b) LSS improved to nine 10 -second holds.

Physiotherapy sessions continued twice weekly with similar treatment. Muscle lengthening techniques were more vigorous and taken further into range. A holdrelax proprioceptive neuromuscular facilitation technique was added for each specific tight muscle group (Janda 1988). Postural re-education was commenced and correct neutral posture was taught in sitting and standing. Visual and sensory awareness of the new corrected posture was provided.

\section{His home programme was altered to:}

a) eight 10 -second holds with the chin tuck action.

b) eight 10-second static contractions of the LSS.

c) practising the correct neutral posture at specific times during the day such as in the car, watching TV, in the shower and at school.

d) continuation of the four stretching exercises.

\section{Weeks 5 and 6}

All cervical active movements were full range and pain free with gentle overpressure. A complete vertebral artery test was now performed and revealed no abnormalities. On palpation the left upper fibres of trapezius, scalenes, levator scapulae and the deep cervical extensors no longer felt tight or appeared to be in spasm. The subject's endurance capacity of the:

a) DCF improved to ten 10 -second holds.

b) LSS improved to twelve 10 second holds.
Physiotherapy sessions were reduced to once a week and were similar. A postural correction and reversal exercise in prone described by Jull (1988) was taught to the patient in order to gain good facilitation and co-contraction of the lower scapular stabilisers and the deep cervical flexors (Figure 5).

\section{His home programme was progressed to:}

a) ten 10-second holds with the chin tuck action.

b) ten 10-second holds of the postural correction and reversal exercise.

c) continued practise of the correct neutral posture and muscle lengthening exercises.

\section{RESULTS}

All of the patients symptoms decreased markedly in frequency over the study period. This is clearly depicted in graphical form below (Figure 6).

There was a reduction in intensity of symptom 1 from $2.4 / 5$ in weeks 1 and 2 to $1 / 5$ in weeks 5 and 6 . The mean intensities of symptom 2 and 3 were essentially unchanged.

All cervical ranges of active movement were restored to full range and were painfree with gentle overpressure.

The left UFT, scalenes, levator scapulae, and the suboccipital extensor muscle groups were felt to be of normal length by the end of the study.

The subject's endurance capacity of the DCF improved from four to twelve 10second holds. Similarly LFT improved from five to twelve 10-second holds.

Telephonic follow-up two months later found the patient to have experienced no symptoms since his last physiotherapy session. One year later he was still clear of all symptoms.

\section{DISCUSSION}

The majority of the patient's symptoms following reduction and immobilisation were felt to be due to:

a) slight persistence of the torticollis causing soft tissue shortening and muscle spasm on the left side.

b) marked weakness and instability due to pain inhibition, ligamentous deficiency and prolonged immobilisation.

The above factors created a muscle imbalance of the "proximal crossed syndrome" type described by Janda (1988) and treatment was therefore aimed at restoring muscle balance.

The results show that a six week cervical stabilisation programme, in a child following reduction of the subluxation, led to a significant improvement in the 

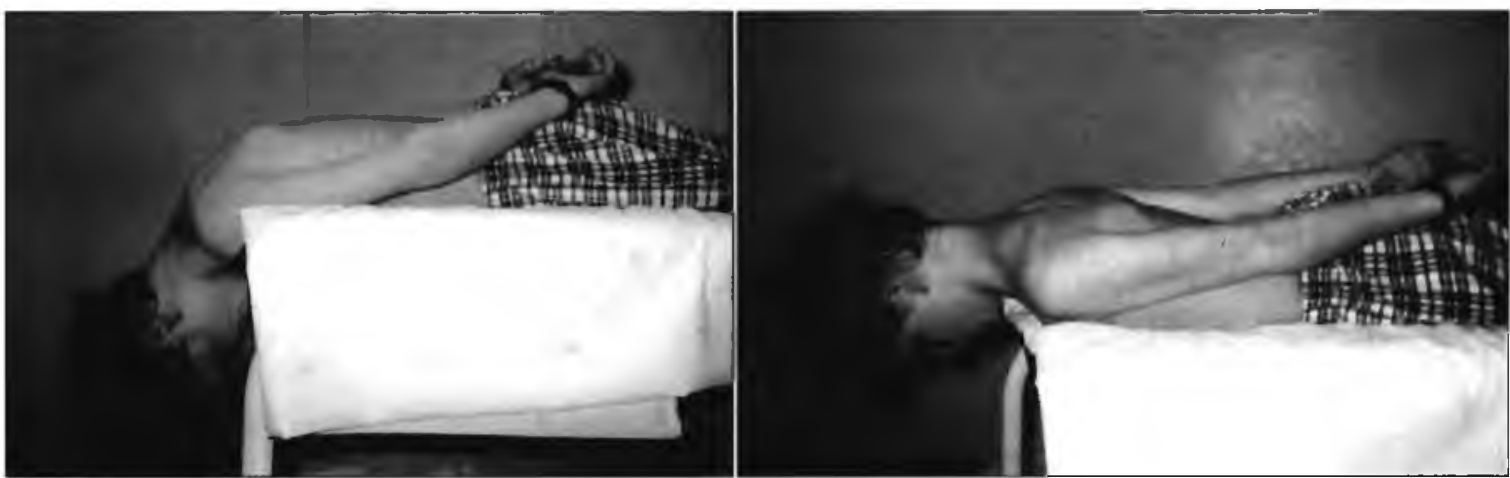

Figure 5

Postural correction and reversal exercise signs and symptoms and an increase in the endurance capacity and lengths of the relevant muscle groups. There was a steady decrease in the frequency of all symptoms with the greatest improvement occurring in weeks 5 and 6.

Unexpected increases in frequency occurred in symptom 1 in week 2 and symptom 3 in week 4 . These were explained on analysis of the subject's diary, which showed returning to school after vacation in week 2 and a tennis clinic in week 4 which were both major provoking factors.

In week 5 the patient reported one "dull, dizzy headache" which appeared to be a reaction to an increase in the number of repetitions of chin tuck holds in his home programme. This reflects the irritability of the upper cervical region and the fact that care needs to be taken in introducing or increasing muscle re-education techniques (Beeton \& Jull, 1994; Janda, 1988). Symptom 3 was the only other symptom reported in weeks 5 and 6 and only occurred in physiotherapy sessions.

There has been no previous attempt to determine the effect of only treating the muscle dysfunction component in patients with chronic cervical pain syndromes (Beeton \& Jull, 1994; Jull, 1988; Janda, 1988). Jull (1988) believes that measures to lengthen muscles and to facilitate muscle activity will not be fully successful unless the articular component is directly treated.

This study has shown, however, that improving the patients muscle dysfunction without directly treating the articular component led to significant reduction of the patient's signs and symptoms.

\section{CONCLUSION}

The presence of an acquired torticollis in a child should arouse suspicion of atlanto-axial rotatory subluxation, particularly where there is a history of a recent URTI or minor trauma. The vital importance of recognition and appropriate referral of this rare condition lies in the fact that it may indicate a compromised atlanto-axial complex, with the potential to cause neural damage or even death.

This study has clearly shown that following reduction of the subluxation, a cervical stabilisation training programme that excluded direct treatment of the articular component, was highly effective in reducing the patient's signs and symptoms. It therefore supports and highlights the important concept of correcting the muscle dysfunction component in cervicogenic pain sufferers.

\section{REFERENCES}

Aspinall W. 1990 Clinical Testing for the Craniovertebral Hypermobility Syndrome.

The Journal of Orthopaedic and Sports

Physical Theropy 12:47-54

Beeton K, Jull G. 1994 Effectiveness of

Manipulative Physiotherapy in the

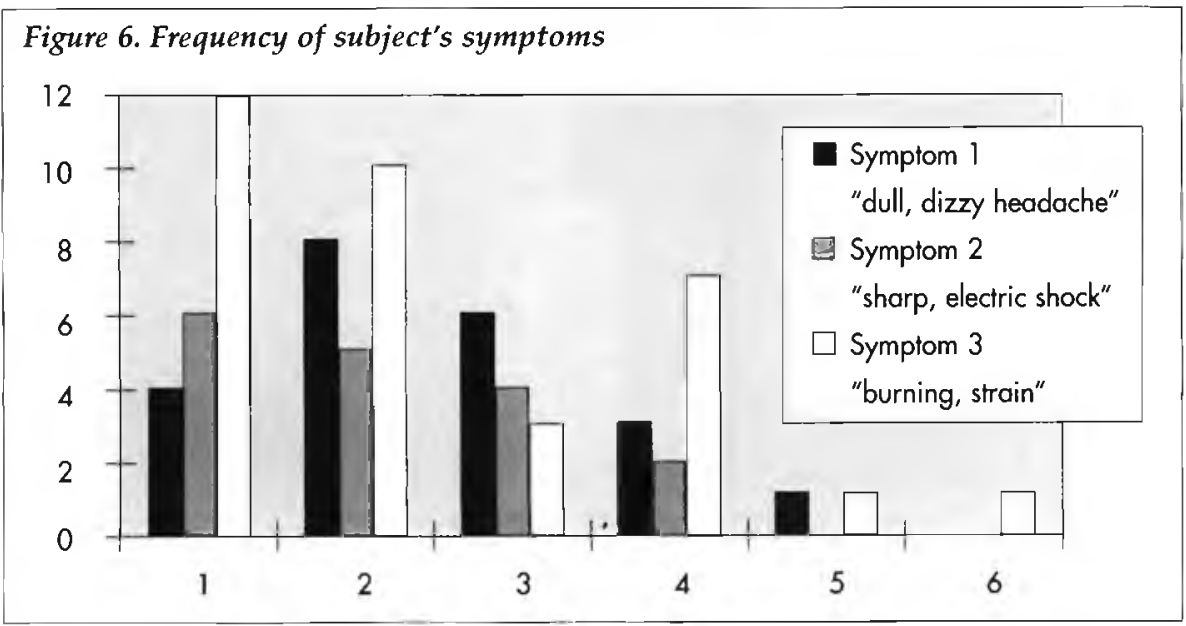

Management of Cervicogenic Headache: A Single Case Study. Physiotherapy 80: 417423.

Bogduk N. 1986 Cervical Causes of Headache and Dizziness. In: Grieve GP (ed) Modern Manual Therapy of the Vertebral Column, pp289-302 Edinburgh: Churchill Livingston.

Dvorak J, Panjabi M. 1987 Functional Anatomy of the Alar Ligaments. Spine 12:183-189.

Edeling J. 1982 The True Cervical Headache. South African Medical Journal 62:531-534. Fielding JW, Hawkins RJ. 1977 Atlanto-

Axial Rotatory Fixation. The Journal of Bone and Joint Surgery 59A:37-44.

Fielding JW, Stillwell WT, Chynn KY, Spyropoulos, EC. 1978 Use of Computed Tomography for the Diagnosis of AtlantoAxial Rotatory Fixation. The Journal of Bone and Joint Surgery. 60A:1102-1104.

Henrys P, Lyne D, Lifton C, Salciccioli, G. 1977 Clinical Review of Cervical Spine Injuries in Children. Clinical Orthopaedics. 129:172-176.

Janda V. 1988 Muscles and Cervicogenic Pain Syndromes. In: Grant R (ed) Physical Therapy of the Cervical and Thoracic Spine, pp153-166. New York: Churchill Livingston. Jull G. 1988 Headaches of Cervical Origin. In: Grant R (ed). Physical Therapy of the Cervical and Thoracic Spine, pp195-218. New York: Churchill Livingston.

Jull G, Richardson C, Hamilton C. 1995 Stabilisation Training for the Cervical Region. Video by the Dept Physiotherapy, University of Queensland.

Phillips WA, Hensinger RN, Arbor A. 1989 The Management of Rotatory Atlanto-Axial Subluxation in Children. The Journal of Bone and Joint Surgery. 71A:664-668.

Roach JW, Duncan D, Wenger DR, Maravilla A, Marivilla K. 1984 Atlanto-Axial Instability and Spinal Cord Compression in Children: Diagnosis by Computerised Tomography. The Journal of Bone and Joint Surgery. 66A:708714.

Robert N, Hensinger RN. 1993 Cervical Spine Problems in Children: Upper Cervical Instability. Transactions of the College of Medicine of South Africa. 41A:45-57.

Watson DH. 1990 Cervical Headache: An Investigation of Natural Head Posture and Upper Cervical Flexor Muscle Performance. In: Grieve GP ( $2^{\text {nd }}$ ed) Modern Manual Therapy of the Vertebral Column, pp249-360. Edinburgh: Churchill Livingston. 